\title{
Copper enhanced nasal saline irrigations: a safe potential treatment and protective factor for COVID-19 infection?*
}

\author{
Thomas Radulesco 1,2, Jerome R. Lechien 1,3-5, Carlos M. Chiesa-Estomba',6, \\ Leigh J. Sowerby ${ }^{1,7}$, Claire Hopkins ${ }^{8}$, Sven Saussez ${ }^{3,9}$, Justin Michel ${ }^{1,2}$ \\ 'COVID-19 Task Force of the Young-Otolaryngologists of the International Federations of Oto-rhino-laryngological Societies \\ (YO-IFOS) \\ ${ }_{2}^{2}$ Aix Marseille Univ, APHM, IUSTI, La Conception University Hospital, Department of Oto-Rhino-Laryngology Head and Neck \\ Surgery, Marseille, France \\ ${ }^{3}$ Department of Human Anatomy and Experimental Oncology, Faculty of Medicine, UMONS Research Institute for Health Scien- \\ ces and Technology, University of Mons (UMons), Mons, Belgium \\ ${ }^{4}$ Department of Otolaryngology - Head and Neck Surgery, Foch Hospital, School of Medicine, UFR Simone Veil, Université Versail- \\ les Saint-Quentin-en-Yvelines (Paris Saclay University), Paris, France \\ ${ }^{5}$ Department of Otorhinolaryngology and Head and Neck Surgery, CHU de Bruxelles, CHU Saint-Pierre, School of Medicine, \\ Université Libre de Bruxelles, Brussels, Belgium \\ ${ }^{6}$ Department of Otorhinolaryngology-Head and Neck Surgery, Hospital Universitario Donostia, San Sebastian, Spain \\ ${ }^{7}$ Department of Otolaryngology - Head and Neck Surgery, University of Western Ontario, London, Ontario, Canada \\ ${ }^{8}$ King's College, London, United Kingdom \\ ${ }^{9}$ Department of Head and Neck Surgery, EpiCURA Hospital, Hornu, Belgium
}

Rhinology Online, Vol 3: 87 - 88, 2020

http://doi.org/10.4193/RHINOL/20.050

*Received for publication:

May 29, 2020

Accepted: June 1, 2020

Published: June 6, 2020

Key words: Nasal lavage, SARS-CoV-2, viruses, viral load, saline, coronavirus infections, pandemics

\section{To the Editor:}

The nasal epithelium has been recognized as an important site of SARS-Cov-2 viral entry and replication ${ }^{(1)}$. Decreasing viral titers using nasal saline irrigation (NSI) may potentially improve patient outcomes and reduce risk of transmission.

Since the outbreak of the COVID-19 pandemic, there has been much discussion regarding the potential use of povidone-iodine to reduce viral load and therefore potentially reduce the risk of transmission and improve patient outcomes. Ramezanpour et al. recently reported on the in vitro safety of $0.5 \%$ povidone-iodine (Nasodine), with consideration for use in chronic rhinosinusitis (2). While contact with Nasodine for five minutes appeared to cause no harm, contact for 30 minutes in vitro was found to impact the epithelial barrier structure, highlighting the need for further studies regarding safety. Nasodine is not commercially available outside of Australia, instead many centers are advocating use of diluted solutions of povidone-iodine, but this carries risk of error, which may cause harm; Kim et al. demonstrated that a $5 \%$ povidone-iodine solution had a significant and immediate effect on reducing ciliary beat frequency to zero ${ }^{(3)}$.

In contrast, nasal saline irrigation (NSI) is a widely used interven- tion with established safety and tolerability. The use of NSI in COVID-19 remains controversial due a theoretical risk of increased pulmonary dissemination, however there is no published evidence to support this. Indeed, we believe that NSI in itself may be beneficial in COVID-19.

NSI may decrease viral loads in nasal cavities during rhinovirus infection, and viruses can be collected when performing NSI, suggesting a "viral washing-effect" (4). Recent computational fluid dynamics studies reported that all nasal regions are reached when performing NSI, particularly using large-volume irrigation versus continuous spraying ${ }^{(5)}$.

NSI may also enhance epithelial function. In vitro studies have demonstrated that an isotonic solution with slightly alkaline $\mathrm{pH}$ and a composition close to that of sea-water optimizes trophic and functional recovery of the respiratory epithelium ${ }^{(6)}$. In chronic rhinosinusitis, NSI improves mucociliary clearance ${ }^{(7)}$, allowing removal of pathogens trapped by local IgA. NSI and oral rinse are commonly used in upper respiratory tract infections and have demonstrated superiority regarding duration of illness versus standard care in a randomized controlled trial ${ }^{(8)}$.

Furthermore, several viruses, including human coronavirus $229 \mathrm{E}$ 
(HCoV-229E), are inhibited in the presence of $\mathrm{NaCl}$. Ramalingam et al. recently showed that patients using NSI had statistically significant decreased household transmission and viral shedding ${ }^{(8)}$. Similarly, nasal sprays and irrigations have been shown to reduce the Influenza A viral load in nasal secretions and enhance mucosal barrier function ${ }^{(9)}$. Hendley et al. found virus concentrations return to baseline 5 days after saline NSI in rhinovirus infections ${ }^{(4)}$.

We were delighted to find the recent report of Huang et al, describing the in vitro anti-viral efficacy of a widely available, copper enriched saline solution, Sterimar Congestion relief ${ }^{(10)}$. Although hypertonic, which may cause slight nasal irritation, this solution may have the benefits of povidone-iodine but without the risk of toxicity. It is commercially available in many countries and has been widely used without reports of adverse effects. Further more, a recent study in the New England Journal suggests that copper was able to eliminate all viable SARS-Cov-2, while the virus remained viable on a range of other materials ${ }^{(11)}$, suggesting that copper may have a unique role as an antiviral that merits further evaluation.
All measures that control infection and viral shedding would help reduce transmission. No study, to date, has evaluated use of copper enriched nasal saline irrigation in COVID-19 positive patients. However, taken together, these data on saline irrigation, the in vitro study of coper enhanced saline, and the potential benefit of copper itself, suggest that this solution may decrease the potential risk of viral infection by both a mechanical and biological effect. As simple as it sounds, copper enhanced saline may be a very useful adjunct in mitigation of COVID-19 - future trials are needed in COVID-19 to evaluate this further.

\section{Funding source}

None

\section{Conflict of interest}

None

\section{Abbreviations}

NSI: nasal saline irrigations

\section{References}

1. Carrouel F, Conte MP, Fisher J et al. COVID19: A Recommendation to Examine the Effect of Mouthrinses with $\beta$-Cyclodextrin Combined with Citrox in Preventing Infection and Progression. J Clin Med. 2020;9(4):1126.

2. Ramezanpour M, Smith JLP, Psaltis AJ et al. In vitro safety evaluation of a povidoneiodine solution applied to human nasa epithelial cells. Int Forum Allergy Rhinol. 2020;10.1002/alr.22575. doi:10.1002/ alr.22575

3. Kim JH, Rimmer J, Mrad N, Ahmadzada S, Harvey RJ. Betadine has a ciliotoxic effect on ciliated human respiratory cells. Laryngol Otol. 2015;129 Suppl 1:S45-S50.

4. Hendley JO, Gwaltney JM. Viral titers in nasal lining fluid compared to viral titers in nasal washes during experimental rhinovirus infection. J Clin Virol. 2004;30(4):326-328.

5. de Gabory L, Reville N, Baux Y, Boisson N, Bordenave L. Numerical simulation of two consecutive nasal respiratory cycles: toward a better understanding of nasal physiology: Simulation of nasal physiology. Int Forum Allergy Rhinol. 2018;8(6):676-685.
6. Bastier P-L, Lechot A, Bordenave L, Durand M, de Gabory L. Nasal irrigation: From empiricism to evidence-based medicine. A review. Eur Ann Otorhinolaryngol Head Neck Dis. 2015;132(5):281-285.

7. de Gabory L, Escabasse V, Boudard P, de Bonnecaze G, Rumeau C, Jankowski R, et al. Prospective, randomized, controlled, open-label study to compare efficacy of a mineral-rich solution vs normal saline after complete ethmoidectomy. Eur Arch Otorhinolaryngol. 2019;276(2):447-457.

8. Ramalingam S, Graham C, Dove J, Morrice L, Sheikh A. A pilot, open labelled, randomised controlled trial of hypertonic saline nasal irrigation and gargling for the common cold. Sci Rep. 2019;9(1):1015.

9. Leibbrandt A, Meier C, König-Schuster M, Weinmüllner R, Kalthoff D, Pflugfelder B, et al. Iota-Carrageenan Is a Potent Inhibitor of Influenza A Virus Infection. Park M-S. PLoS ONE. 2010:5(12):e14320.

10. Huang S, Constant S, De Servi B, Melon M, Culig J, Bertini $M$, et al. In vitro safety and performance evaluation of a seawater solution enriched with copper, hyaluronic acid, and eucalyptus for nasal lavage. Med
Devices Auckl NZ. 2019:12:399-410.

11. van Doremalen $\mathrm{N}$, Bushmaker $\mathrm{T}$, Morris $\mathrm{DH}$ Holbrook MG, Gamble A, Williamson BN, et al. Aerosol and Surface Stability of SARSCoV-2 as Compared with SARS-CoV-1. N Engl J Med. 2020;382(16):1564-1567.

Dr Thomas Radulesco, MD, PhD, MS Department of Oto-Rhino-Laryngology

Head and Neck Surgery

La Conception University Hospital

147 Bd Baille

13005 Marseille

France

Tel: +33491435580

Fax: +33491435810

E-mail: thomas.radulesco@ap-hm.fr

ORCID ID: 0000-0002-5939-5372 